\title{
TARNATION OU LA VIE EN JEU
}

\author{
Lise Gantheret ${ }^{1}$
}

Film à teneur autobiographique retraçant trente ans de la vie de son réalisateur, Tarnation de Jonathan Caouette, illustre clairement l'individualisme expressif actuel en régime médiatique. Par ce film, constitué d'archives familiales tournées en Hi8, Super 8, Betamax, VHS, DV et assemblées sur un Macintosh à l'aide du logiciel iMovie, Caouette tente de comprendre la déchéance schizophrène de sa mère tout en tachant de se trouver lui-même. Empruntant à la sémio-pragmatique et à la psychanalyse, Lise Gantheret examine comment les techniques audiovisuelles rendent explicites cette idée fondamentale de l'alliance entre la forme et le fond dans l'expression de soi. Plus spécifiquement, elle montre comment la construction identitaire est corroborée par la nécessité d'une certaine mise en scène de soi alliant images et discours sur soi (son et écriture) que ce soit par la performance de soi, l'autoreprésentation, la remédiation ou encore l'autofiction. Elle met ainsi en lumière une véritable thérapeutique identitaire poursuivie par le réalisateur qui s'af-

1 Cine Institute, Jacmel, Haiti. Cet article est issu d'une communication intitulée « Tournage-Montage d'une identité à l'ère des nouvelles technologies audiovisuelles. L'exemple de Tarnation (2004) de Jonathan Caouette » faite dans le cadre du 77ème congrès de l'ACFAS sur le thème « Les techniques de soi à l'ère des technologies de l'information et de la communication », Université du Québec à Montréal, 2009.

Recherches en communication, $\mathrm{n}^{\circ} 36$ (2011). 
firme par la visibilité et la suture. Ce dernier peut ainsi aboutir à sa reconnaissance tant sur le plan physique, psychique, social que spirituel. Subséquemment, ce film tend à prouver que loin d'asservir, ces nouvelles techniques, désormais plus facilement accessibles à tout un chacun et miniaturisées, favorisent des processus d'émancipation et d'individuation ou encore servent d'exutoire cathartique pour se connaître et se construire soimême en vue d'une meilleure adéquation au monde.

Le film autobiographique Tarnation (2006) de Jonathan Caouette ${ }^{1}$ retrace 30 ans de traumatismes psychiques liés à des dysfonctionnements familiaux. Sa mère, devenue schizophrène suite à une chute d'un toit à l'âge de 13 ans, sera dans l'incapacité de s'occuper de son fils alors que son compagnon partira sans donner de nouvelles. Pris en charge par ses grands-parents, puis par une famille d'accueil, Caouette sera victime de maltraitance. Néanmoins, dès son plus jeune âge il remédie à la défaillance parentale grâce à l'autofilmage. Enjeux de vie, d'écriture et de représentation s'interpénètrent.

Dans cette perspective, la pratique filmique autobiographique s'apparente à une technique de soi permettant à son auteur de pallier le chaos familial afin de rendre possible les ajustements identitaires nécessaires à la structuration de soi. Ce film appartient aux « nouvelles productions mémorielles » qui «n'hésitent pas à faire apparaître les aspects de la vie de famille qui jusque-là restaient dans le non-dit » (Odin, 2011, p. 95). Il s'inscrit également dans cette large production de films personnels qui mettent en œuvre une forte énonciation personnelle ${ }^{2}$ (Gantheret, 2011).

Par une approche empruntant à la psychanalyse, à la philosophie foucaldienne et ricœurienne et à la sémio-pragmatique, nous souhaitons montrer comment cette production filmique liée aux technologies audiovisuelles permet à Jonathan Caouette de prendre soin de lui et de se façonner un discours identitaire fort en fonction des dispositifs spécifiques du medium utilisé, des remédiations ou reprises médiatiques pratiquées, et des espaces de communication convoqués.

1 Ce jeune cinéaste a filmé son journal de bord de l'âge de 9 ans à 24 ans, et a ensuite procédé à son montage avec le logiciel iMovie.

2 «Par énonciation personnelle, nous entendons toute trace de la personne dans l'énoncé, ou encore tout signe qui renvoie à ce 'qui porte la marque nettement accusée du caractère, des idées et des goûts de quelqu'un'» (Gantheret, 2011, p.101, sur base d'une définition du dictionnaire Larousse). 


\section{Les enjeux de l'auto-représentation}

Tout d'abord il convient de situer ce film dans le contexte particulier d'une société où " l'individualisme expressif » (Allard, 2011) est de plus en plus prégnant et les technologies audiovisuelles largement démocratisées. Comme le souligne Allard en citant Ulrich Beck, Charles Taylor et Anthony Giddens, force est de constater

[...] ce moment contemporain d'individualisation plus réflexif au sujet de «qui voulons-nous être? ?. Leur diagnostic est qu'en raison du processus de détraditionnalisation des grandes institutions pourvoyeuses d'identités, comme la famille, le salariat, etc., les identités personnelles et sociales ne sont plus données naturellement ni reproduites aveuglément. Elles deviennent en partie, la résultante d'un travail expressif à travers lequel l'individu façonne sa biographie et se dote d'une identité certaine (Allard, 2011, p. 66).

La démarche de Jonathan Caouette consiste effectivement à répondre à la question " Qui suis-je ? » par le biais de la performance et de la reconstitution filmique. À cette fin, il use de l'autoreprésentation : il se filme fréquemment dans des lieux clos face à la caméra dans des poses intimistes. De ce dispositif résulte ce que nous appelons une image indice de soi, c'est-à-dire l'image d'un autre soi-même matérialisé visuellement (photographie, film, vidéo). Ce moment de soi historisé et réifié peut alors servir d'outil d'interrogation du sujet pour mieux se définir. Se rejouerait le stade du miroir (Lacan, 1966), soit le moment où l'enfant va prendre conscience de sa forme corporelle et du jeu des illusions. Au montage, l'insertion de nombreuses photos ou documents audiovisuels représentant le sujet participent de ce même processus de définition de soi.

Ce recours marqué à l'autoreprésentation et à l'autopublicisation semble trouver plus spécifiquement son origine dans l'expérience médiatique de la mère de Jonathan Caouette, Renée. Adolescente, elle est en effet choisie comme modèle photographique. Immergée dans le milieu de la publicité, elle est fortement influencée par la société du paraître : Elisabeth Taylor et les comédies musicales qu'elle imite sont ses seuls référents. Par mimétisme identifiant et imprégné d'une culture de l'image (télévisuelle et cinématographique), Jonathan Caouette emprunte un modèle similaire de construction de soi. Par exemple, il se 
filme dans de petites mises en scène théâtrales ou bien chante en playback sur des films musicaux. À propos de ce type de productions, Roger Odin souligne qu'elles fonctionnent sur un mode spécularisant dont la spécificité est de renvoyer à un énonciateur réel. C'est-à-dire que même si l'espace est imaginaire, la relation affective au sujet renvoie le spectateur à la performance d'un être réel, soit au comédien. Subséquemment, par ce biais, Caouette afficherait une identité et une vocation d'acteur (ce qu'il deviendra effectivement).

L'autofilmage apparaît également comme la nécessité pour Jonathan Caouette de se donner à voir pour exister. En effet, cette démultiplication formelle de soi peut être lue comme une difficulté à s'inscrire au monde. La mise en abîme spéculaire de son image souligne la part fantomatique et labile du soi notamment en raison de l'emprise maternelle. «Tu n'es rien sans moi », dit-elle. Cette parole relie irrémédiablement la vie de Jonathan à sa mère. «Elle est partout en moi, à l'intérieur de moi », constate-t-il au final du film. La relation entre Caouette et sa mère serait en partie comparable à celle d'un enfant in utero qui ne connaît de la réalité que le réel de l'unité avec la mère. Cette homogémellité implique une difficile différenciation entre le dedans propre et le dehors maternel. D'un point de vue formel, les nombreux cadrages où mère et fils apparaissent indissociables, traduisent cet état de fait. L'usage d'images combinatoires figure le constat de cette fusion mais révèle en même temps un désir de séparation. Comment rejeter hors de soi cette part du parent sans se rejeter soi-même, ni le parent lui-même?

En outre, comme l'illustre la présence d'un large éventail de figurations du rapport éros/thanatos, la rupture face à cette relation symbiotique et à cette situation familiale en général est douloureuse. Jonathan Caouette se filme en pleurs dans des situations explosives, les cheveux en bataille, défiant la folie et la mort : "Filming things had a critical life-and-death purpose. It was always a defense mecanism and a way to have a sense of control over my life. Filming was also a way to control and defend myself from the horrors around $m e »^{1}$. Un intertitre du film indique : « Face au chaos et au manque de structure, il fait une tentative de suicide ». Aussi cet exhibitionnisme ou cette surabondance du corps imagé s'avérerait pour Caouette une façon d'accéder au symbolique et de réaffirmer son propre désir narcissique.

1 Dossier pour la presse : Tarnation : un film de - a film by Jonathan Caouette, Montréal, Les Films Séville Pictures, 2003, p. 9. 


\section{Techniques de soi audiovisuelles et enjeux spectatoriels et psychanalytiques}

Le dispositif d'autofilmage peut lui-même participer de cette construction identitaire en dépit d'une certaine limitation : celle de la non-confrontation avec un corps physique humain ${ }^{1}$, qui constitue un manquement ontologique aux prédicats physiques et psychiques constitutifs de l'homme (Ricœur, 1990, p. 46). Car paradoxalement la spécificité de l'autofilmage, par l'impossible entièreté de l'identification dans l'espace de communication en raison de l'absence d'un corps de chair et d'un esprit, renverrait le sujet à la prise de conscience de sa différence, soit justement de son humanité. Ainsi, par ce manque, le filmé ferait l'épreuve de son ipséité, de son soi. En outre, le dispositif technique mis en place offre d'une certaine façon une possibilité d'interlocution : ce positionnement d'un individu face à la caméra s'apparenterait au dispositif psychanalytique, soulignant la nécessité d'une conscience regardante et écoutante.

La comparaison avec la cure psychanalytique est corroborée par la première séquence du film dans laquelle Jonathan Caouette raconte ses rêves comme s'il était sur un divan. Il évoque son cauchemar en compagnie de son petit ami dans son lit. À ce niveau s'entremêlent deux types de discours propres à toute psychanalyse. L'un concerne l'interprétation des rêves dans lequel Caouette explique l'angoisse qu'il vit vis-à-vis de sa mère. Le second a trait à la confession lorsqu'il révèle (par l'image) l'homosexualité de sa relation de couple. Par cette mise en scène, il affirme son mode d'être en tant que sujet social (Foucault, 1984, p. 46).

Cependant, à convoquer la psychanalyse, qu'en est-il de la cheville ouvrière de la cure : le transfert ? La caméra, intercesseur médiatique, ne peut jouer que partiellement ce rôle du psychanalyste par manquement ontologique. Dès lors, la propension des réalisateurs à exposer ces films intimistes dans la sphère publique incite à prendre sérieusement en compte la place du spectateur dans le processus de transfert. L'expérience spectatorielle incluse dans l'espace de communication de la réception induirait pour le filmé une conscientisation partagée de

1 En effet, ce regard externe correspond aussi à l'œil regardant de la caméra, soit à un œil mécanique posé sur un trépied et parfois tenu par un tiers. 
ses traumatismes par le déplacement de sa conduite émotionnelle par rapport à ses parents. En d'autres termes, par sa seule présence, le spectateur $^{1}$ validerait la souffrance du cinéaste, ouvrant par ce transfert vers un processus de guérison de ce dernier.

Pour autant, Jonathan Caouette n'épargne pas au spectateur une implication parfois dérangeante voire tyrannique de son intimité (Sennett, 1979). La caméra placée extrêmement proche du sujet peut procurer une impression de promiscuité envahissante démultipliée par le fait que ce film ne fonctionne pas sur un mode fictionnalisant mais documentarisant. En effet, malgré la retranscription hallucinogène de son expérience, les réalités de l'énoncé et de l'énonciateur ancrent plus instamment le spectateur dans un mode de lecture documentarisant (Odin, 2000, p. 127), notamment par la narration via les intertitres qui documentent la biographie de l'auteur. En conséquence, le spectateur est interpelé plus fortement en tant que personne réelle. D'ailleurs, Caouette dit vouloir faire ressentir « un choc cardiaque massif » (Roy, 2005, p. 40) au sens littéral et figuré. Sa mère, diagnostiquée schizophrénique bipolaire, a reçu des traitements aux électrochocs dans les institutions psychiatriques du Texas entre 1960 et 1970. Ces derniers n'ont fait qu'accentuer les symptômes. Le réalisateur n'hésite pas à signifier ces désordres psychologiques par des jeux de montages stroboscopiques, dont le choc visuel veut imiter la choc cardiaque : « J'ai voulu que le spectateur éprouve ce que c'est de vivre avec la maladie mentale, ce qui peut être enfantin et déstabilisant à la fois » (Roy, 2005, p. 39).

Les prises de vue ne se réduisent pas à un autofilmage mais s'étendent aux proches de Jonathan Caouette, notamment sa mère. D'un côté, l'acte de filmer peut être vecteur de protection : " J'avais l'impression que la caméra était comme une arme ou un bouclier, dit-il, une manière de garder le contrôle sur ce que je traversais » (Rigoulet, 2004b, p. 28). D'un autre côté, cependant, l'acte de filmer ouvre pour Caouette de nouveaux espaces et rapports communicationnels au sein de sa famille. Cette technique de soi «n'est pas un exercice de solitude, mais une véritable pratique sociale ». (Foucault, 1984, p. 62). À ce titre, la caméra devient un outil relationnel et d'intervention immédiate sur le réel. Il s'agit tout à la fois d'un mode d'investigation (Jonathan interroge ses grands-parents sur son passé familial) mais aussi de compré-

1 Notons que le cinéaste est souvent son premier spectateur. 
hension de soi et des autres dans leurs histoires de vie respectives. Par exemple, Caouette révèle un souci de soi corrélé à un souci constant pour sa mère.

Mais il arrive aussi que le jeu entre le soin de soi et l'aide de l'autre s'insère dans des relations préexistantes auxquelles il donne une coloration nouvelle et une chaleur plus grande. Le souci de soi - ou le soin qu'on prend du souci que les autres doivent avoir d'eux-mêmes - apparaît alors comme une intensification des relations sociales (Foucault, 1984, p. 74).

Filmer l'autre s'avère même vecteur d'union. Le film de Caouette sera prétexte à cette scène significative : la réunion de ses parents dans son appartement new-yorkais. Le cinéaste trouve ainsi la filiation manquante : la rencontre avec son père a été motivée par son projet de film.

\section{Thérapeutique identitaire du discours sur soi}

L'acte de filmer constitue donc un premier outil de subjectivisation permettant à Jonathan Caouette d'élaborer son propre discours sur le monde. Par la suite, le montage amplifiera ce discours intimiste et réflexif, ouvrant sur une réconciliation de soi avec soi. C'est à ce stade que se constitue l'identité narrative du sujet grâce à un récit réorganisant l'expérience temporelle. Le premier geste biographique de Caouette sera de faire un point générationnel. D'emblée, il instaure un récit de vie de l'ordre de la fable : «Il était une fois dans un village au Texas ». Le mode documentarisant se mêle au mode fabulisant. Des intertitres explicatifs narrés à la troisième personne convoquent une subjectivité indirecte instaurant une distanciation des affects et assurent une cohérence dans le discours du sujet.

En effet, ce film permet de canaliser les perturbations émotionnelles en les combinant dans une tension narrative. La rhétorique des affects s'énonce à travers les séquences filmées tandis que la rhétorique historisante tient des intertitres à vocation biographique. Se perçoit le désir de préserver une structure classique dans cette reconstitution biographique à base d'archives' ${ }^{1}$. Toutefois, la non-linéarité du flux visuel, métaphore des perturbations émotives, s'oppose à la chrono-

1 Caouette dispose de 160 heures de matériel- (Roy, 2005, p. 40). 
logie biographique ${ }^{1}$ qui constitue l'axe identitaire de ce récit filmé de vie.

Car outre le contexte familial, une des difficultés de l'auteur à se situer au monde provient d'un autre problème psychologique. Jonathan Caouette est victime de troubles dissociatifs suite à l'ingestion d'une drogue forte à l'âge de 12 ans. Par le biais d'effets de duplication, où chaque image vient modifier la modalité de production et d'identification, Caouette interroge cette dualité de l'être. D'ailleurs nombreuses sont les photographies d'identité à être reproduites et démultipliées. À travers ces répliques imagées, le réalisateur questionne les standards de représentation et ce que nous appelons l'image convenue de soi. Mais la réplique au sens d'Eco $(1992$, p. 14) n'est jamais absolue et la reproductibilité du corps humain renvoie seulement à une réplique partielle. Aussi, accentuant cette idée d'une définition de soi par une confrontation à une pluralité hétérogène, Caouette recourt à un discours intermédial fort de l'ordre de la reprise. Il mêle des extraits de journaux filmés, des films de famille, de reportages télévisés, de longs-métrages de fiction, d'anciens messages téléphoniques, des photos, ou encore des affiches. Les relations intertextuelles entre son film et ceux d'autres cinéastes suggèrent le besoin de se comprendre soi-même par la comparaison avec d'autres discours. Subséquemment, grâce à l'entremise des technologies audiovisuelles, chaque image avance une renégociation identitaire avec la précédente pour se préciser vers une mise au point. Évitant toute standardisation du récit filmique et du flux visuel, Caouette procède ainsi à la constitution imagée de son identité. Cet assemblage se proposerait alors comme curatif à cette eternal damnation - contraction de Tarnation en texan.

J'ai su avant même de monter le film que ce dont je disposais m'impliquait violemment et il a fallu me dissocier de tout cela, ne serait-ce que pour pouvoir travailler convenablement ce matériel et éviter toute complaisance (Roy, 2005, p. 40)

Pour Caouette, l'enjeu réside donc dans la construction progressive de son individualité psychique par le discours, lorsque le JE (de l'auteur) pourra être différencié du TU (de la mère et des autres) via le IL (son soi imagé).

1 On observe malgré tout une redondance de certaines séquences fortes, parflashbacks, qui insistent continuellement sur l'incidence du passé dans le présent. 


\section{Déchiffrement et affirmation de soi : de la cellule familiale à la famille artistique}

L'autobiographe possède dès lors un pouvoir de compréhension et d'historisation sur le défilement de sa vie. En ce sens, la pratique vidéo favorise l'entendement de la destinée d'un sujet par un possible contrôle temporel et mémoriel (constitution d'archives visuelles consultables) de sa vie. Reproductibilité et conservation des données offrent à Jonathan Caouette la capacité d'user du film comme procédé mnémotechnique.

Si se convertir à soi, c'est se détourner des préoccupations de l'extérieur, des soucis de l'ambition, de la crainte devant l'avenir, on peut alors se retourner vers son propre passé, en faire la recollection, le dérouler à son gré sous ses propres yeux et avoir sur lui un rapport que rien ne viendra troubler (Foucault, 1984, p. 91).

Dans ce processus de reconstruction de la mémoire familiale, Jonathan Caouette compose son discours non seulement sur le mode intime ${ }^{1}$ mais aussi sur le mode témoignage (Odin, 2011, p. 97). Il fait ainsi s'équivaloir, par le cadrage, son autoportrait avec les portraits et interviews de ses proches, instaurant un processus dialectique de construction de soi. Le solipsisme du dispositif d'une caméra placée face à soi, qu'illustrent les séquences d'autoportraits, est ainsi contrebalancé par l'insertion d'images de tiers ou réalisées par des tiers (sa mère, son père, ses grands-parents, lui-même à différents stades de sa vie, des films d'autres personnes, etc.). Comme le souligne Odin (2011, p. 97), dans ce type de production, « on est loin de la production de consensus ». Les tensions familiales sont explicitées. Se révèlent alors « les vieilles rancœurs, les vieux conflits entre les membres de la famille, tout le non-dit qui constitue la face d'ombre de la famille» (Odin, 2011, p. 97).

D'ailleurs, en revisitant un film de famille tourné dans l'une de ses familles d'accueil, le réalisateur met en lumière des vérités qui contre-

1 « Le mode intime se manifeste sous la forme d'un discours intérieur : il n'y a pas extériorisation de la communication. Inutile d'insister sur la force des affects qui animent cette production interne et son rôle sur la construction identitaire de l'individu, une construction qui se fait par différenciation par rapport aux autres » (Odin, 2011, p. 86). 
disent celles, idylliques, affichées par l'institution familiale. Par une nouvelle recontextualisation, via l'usage du ralenti, le réalisateur transforme une gestuelle innocente en acte de perversion. Dès lors, le regard à demi endormi de jeune enfant qu'était Jonathan Caouette, et qui aurait pu signifier la douceur d'une enfance protégée, peut être lu désormais comme celui d'un être prisonnier. Cette migration du film de famille en dehors de son contexte d'origine a pour but de lui faire prendre un sens nouveau. Tarnation consisterait en un exercice de conscientisation (au risque de la victimisation) qui se veut triple : celle de soi-même, celle de son bourreau et celle du tout sociétal, lequel valide la maltraitance par masquage délibéré ou par aveuglement et impuissance. Ce film est l'occasion pour Caouette de communiquer ses valeurs. En ce sens, Caouette mobilise le mode moralisant car il s'interroge non seulement sur son identité mais aussi sur son action au monde et les valeurs qui la motivent. La présence dans la première séquence du film d'une voix de femme qui lit un texte ${ }^{1}$ proposant quelques leçons de vie sur le maintien de soi, métaphore d'une voix angélique ou de la conscience de Caouette, corrobore cette nécessité de se donner un guide éthique.

La convocation de ce mode moralisant implique que «l'énonciateur réel soit interrogeable en termes d'identité, de faire et de valeur » (Odin, 2011, p. 58). Toutefois, cette évaluation moralisante, lorsqu'elle est assignée au film de Caouette, révèle que des faits historiques comme la paternité 2 de ce dernier (Rigoulet, 2004a, p. 37) sont totalement oblitérés du film. La raison tient aux exigences de la production « tombées » ultérieurement sur le projet de rendre le film plus percutant. Aussi, l'éviction de certains actants de la mémoire familiale, comme le propre fils de Caouette, révèle le désir d'assigner à ce dernier la place du fils et non celle du père, comme s'il fallait accentuer la relation œdipienne qu'il entretient avec sa mère. Bien qu'expression de soi, ce film reste

1 «Allez tranquillement parmi le vacarme et la hâte et souvenez-vous de la paix qui peut exister dans le silence. Sans aliénation, vivez, autant que possible en bons termes avec toutes les personnes. Dites doucement et clairement votre vérité. Écoutez les autres, même les simples d'esprit et les ignorants, ils ont eux aussi leur histoire. Évitez les individus bruyants et agressifs, ils sont une vexation pour l'esprit » (extrait d'un texte trouvé dans la Cathédrale Saint-Paul de Baltimore en 1692, auteur inconnu).

2 « Dès qu'on entre dans les cercles de la production et de la distribution, ce sont des discussions et des conflits sans fin. J'ai fini par me laisser convaincre que le film gagnerait à être simplifié, mais on a coupé des pans entiers de ma vie, comme l'histoire de mon fils de 9 ans par exemple » (Rigoulet, 2004a, p. 37). 
donc sous la contrainte de l'industrie cinématographique, avec son espace de production et de distribution.

Reste que le film de Jonathan Caouette appartient au secteur indépendant de la production, voire à un cinéma de la marge. Plus encore, Caouette vise à rejoindre l'espace de l'art en faisant de son film un manifeste expérimental homosexuel. La recherche d'une homologie structurelle avec les films du milieu underground (notamment les films punks, gay et expérimentaux du milieu new-yorkais) qu'il fréquente depuis son adolescence s'inscrit dans cette quête identitaire et rejoint son besoin d'affirmation en tant qu'artiste gay. C'est pourquoi ce film mobilise fortement le mode artistique. Sur le plan énonciatif, Caouette asserte son nom en citant ses propres court-métrages ${ }^{1}$ ( $L a$ cheville brisée, Sale pute et Garçon de salive et de sang) au travers desquels il mène diverses expériences transgressives vis-à-vis des conventions mondaines et normatives. Il renouvelle ainsi un travail sur la réminiscence, la nostalgie et la souffrance : drogue, sexe et rock and roll sont affichés sans retenue. Il revendique même explicitement sa filiation : «Ce pourrait être un film culte underground » (Roy, 2005, p. 40). Au niveau discursif, il rappelle ses références à la rhétorique des films expérimentaux par la copie de plans, par l'imitation de cadrage, par l'usage de l'irisation ou encore par le jeu d'effets d'irréalité et de travestissement. Le journaliste Charles-Stéphane Roy décrit avec justesse l'impact spectatoriel de ces effets :

On ne sort pas indemne de Tarnation. [...] Physiquement on suit un bombardement rétinien en règle alors que l'écran nous matraque sans cesse d'une imposante masse d'informations morcelée et déconstruite. Émotivement, on est saisi par de nombreuses scènes qui giflent l'épiderme (Roy, 2005, p. 39).

Outre l'espace de l'art, c'est un espace de vie que Jonathan Caouette souhaite créer. Ainsi, lorsque le réalisateur use d'effets visuels et rythmiques multiples (dissection, détournement, surimpression mais aussi découpe de l'écran en plusieurs fenêtres) pour évoquer ses errances et ses troubles de dépersonnalisation, il mobilise un autre mode : le mode énergétique. Selon Odin, ce mode induit des relations fondées sur des

1 Inspirés par les films de Jim Jarmush et de Georges Roméro. On perçoit également l'influence des films de David Lynch, Paul Morissey, Andy Warhol et Gus Van Sant, ainsi que de la comédie musicale Hair (1979). 
effets plus que sur des affects : «Dans ces moments, il ne s'agit plus de me faire vibrer au rythme des événements racontés (mis en phases) mais de me faire vibrer au rythme des images et des sons. Les affects cèdent la place aux effets » (Odin, 2011, p. 52). Peut-être est-ce une manière pour Caouette de conjurer ses pulsions suicidaires par une réénergisation vibratoire.

\section{Conclusion}

Toute autobiographie n'est jamais qu'un essai pour s'atteindre soi-même, un mode de production de sens, en l'occurrence de sa vie. Jonathan Caouette s'est inventé sa propre règle de production de soi en jouant d'une fonction sémiotique modulable, offrant sans cesse l'instauration de nouveaux modes de production de sens. C'est par cet examen déconstructiviste qu'il tache de repérer les constantes et de trouver l'unité. Les multiples potentialités d'expression offertes par les techniques filmiques sont employées pour atteindre cette connaissance de soi. Trentenaire, Caouette est désormais capable d'avoir en main les différents éléments qui constituent le puzzle de sa vie, et de déceler les tenants et les aboutissants d'une destinée pour enfin tâcher d'y avoir une prise. L'affirmation de soi s'imposerait alors par la répétition et l'accumulation d'images de soi, par l'exposition publique des secrets de famille, ainsi que par le suivi chronologique de l'évolution de sa vie (jouant entre fixation et défilement des événements). La mémoire altérée par les traumatismes infantiles est ainsi reconstituée et la compréhension des événements familiaux possible. L'entendement par le détachement autorise ultimement un processus de résilience.

En ce sens, l'image de soi médiatisée offrirait la possibilité de séparer, de trier, de couper puis de suturer pour se retrouver soi-même. Cet exercice a nécessairement un pouvoir libérateur. D'une part, le pouvoir structurant de la caméra provient de sa position centripète au corps. Elle servirait de preuve d'authentification, de témoin oculaire. D'autre part, centrifuge à la conscience du filmé, la caméra incite à l'exposition de soi et favorise la constitution des idées car elle pousse à la mise en parole, à l'énonciation d'éventuels traumatismes. Le tournage amplifie la conscience du regard dans la perception de l'être au monde et l'appréhension des formes. Le montage, devenu conscience imageante, offrirait la possibilité d'approfondir la connaissance de soi, malgré la restriction propre à toute représentation. Jonathan Caouette 
s'écrit par la cassure au sens de Derrida (1967). La coupure maîtrisée par la suture participe ainsi à la formation d'un être nouveau. Tarnation s'inscrit dans la représentation morcelée du sujet, révélatrice de l'identité post-moderne.

\section{Références}

Allard, L. (2011). Pragmatique de l'Internet mobile. Technologies de soi et culture du transfert. Dans J. Dervin \& Y. Abbas (Éd.), Technologies numériques du Soi et (co) constructions identitaires (pp. 58-81). Paris : L'Harmattan.

Buckland, W. (2009). Introduction : Puzzle plots. Dans W. Buckland (Éd.), Puzzle films: Complex storytelling in contemporary cinema (pp. 1-12). Chichester : Wiley-Blackwell.

Derrida, J. (1967). De la grammatologie. Paris : Éditions de Minuit.

Eco, U. (1992). La production des signes. Paris : La Librairie française [1 1 ère édition Indiana University Press, 1976].

Foucault, M. (1984). Histoire de la sexualité, vol. 3 : Le souci de soi. Paris : Gallimard.

Gantheret, L. (2011). L'énonciation personnelle du film. Les Cahiers Louis-Lumière, (8), 100-108 [Actes du colloque organisé par l'Ecole supérieure d'audiovisuel (ESAV) de l'Université de Toulouse II le Mirail et l'Ecole nationale supérieure Louis-Lumière à l'Abbaye Ecole de Sorèze, Ecole nationale supérieure LouisLumière, Lyon, 2011].

Lacan, J. (1966). Le stade du miroir comme formateur de la fonction du Je telle qu'elle nous est révélée dans l'expérience psychanalytique. Dans J. Lacan, Écrits (pp. 93100). Paris : Seuil. [Communication faite en 1949.]

Odin, R. (2000). De la fiction. Bruxelles : De Boeck Université.

Odin, R. (2011). Les Espaces de communications. Grenoble : Presses Universitaires de Grenoble.

Ricœur, P. (1990). Soi-même comme un autre. Paris : Seuil.

Rigoulet, L. (2004a, 10 novembre). Le « Home movie » explose sur les écrans. Télérama.

Rigoulet, L. (2004b, 26 mai). Jonathan Caouette, sa vie, son œuvre et vice-versa. Télérama.

Roy, C.S (2005, janvier-février), Jonathan Caouette, entretien. Séquence.

Sennett, R. (1979). Les tyrannies de l'intimité. Paris : Seuil. 